\title{
COVID-19: ABC System and LBP-like Function of ORF7a Activate Monocytes to Induce Diabetes
}

\author{
Wenzhong Liu ${ }^{1,2, *}$, Hualan $\mathrm{Li}^{2}$ \\ 1 School of Computer Science and Engineering, Sichuan University of Science \& Engineering, \\ Zigong, 643002, China; \\ 2 School of Life Science and Food Engineering, Yibin University, Yibin, 644000, China; \\ * Correspondence.Wenzhong Liu, liuwz@suse.edu.cn.
}

\begin{abstract}
Diabetes and obesity are important factors that make COVID-19 worse. Lipopolysaccharide activates the natural immune system response in obese and diabetic patients' adipose tissue and increases the risk of susceptibility and severity of COVID-19. In this study, bioinformatics techniques such as domain search and molecular docking were used to study the relationship between the ORF7a protein of the SARS-COV-2 virus and lipopolysaccharide. The results show that the transmembrane protein ORF7a has ABC transporter domains: ATP binding and ABC transmembrane domains. The ABC transport domain of ORF7a transported molecules such as metal ions, heme, nucleosides, amino acids, oligopeptides, sugars, vitamins, salts, alkalis etc.. Moreover, it had a multi-drug efflux function. ORF7a also has lipopolysaccharide synthesized domains. It bound the lipopolysaccharide synthesized by ORF7a to CD14 molecule through lipopolysaccharide-binding protein (LBP) to activate $\mathrm{CD} 14^{+}$monocytes. The extracellular ORF7a with the N-terminus and C-terminus cut off still has the BPI11/LBP domain ("CELYHYQECVR") and two ATP-binding motifs ("HYQEC" and "TYEGNS"). The extracellular ORF7a had a similar function of LBP, binding and activating CD14+ monocytes with the help of two ATP-binding structures. We speculated that cells infected by the SARS-COV-2 virus often used its surface lipopolysaccharides to build expanded barriers to resist $\mathrm{T}$ cells, NK cells, and drugs. More lipopolysaccharides also activated $\mathrm{CD} 14^{+}$monocytes to release various inflammatory factors, damaging adipose and vascular endothelial tissue to induce diabetes and hypertension.
\end{abstract}

Keywords: Lipopolysaccharide; Endotoxin; ABC transporter; ATP binding domain; BPI11 domain; CD14

\section{Introduction}

Diabetes and obesity are susceptible to COVID-19 disease ${ }^{1}$, and are also essential factors in the transition to the worsening of COVID-19 condition $^{2,3}$. COVID-19 makes infected people susceptible to hyperglycemia; high blood sugar modulates immune and inflammatory responses 4 . Obesity is related to the accumulation of macrophages in white adipose tissue (WAT), contributing to the development of insulin resistance. Intestinal flora induces the collection of lipopolysaccharide (LPS)-dependent macrophages in WAT, the damage to systemic glucose metabolism ${ }^{5}$. Therefore, it is vital to study the pathological mechanism of the susceptibility and severity of COVID-19 in patients with diabetes and obesity caused by endotoxin (lipopolysaccharide) ${ }^{6}$ and LPS-dependent macrophage.

Past and inducible endotoxemia is a critical factor in the severity of the COVID-19 outcome. 
Lipopolysaccharide (LPS) is a biological macromolecule secreted by Gram-negative bacteria, the pathogen of respiratory diseases ${ }^{7}$. Lipopolysaccharide is also an outer membrane glycolipid and innate immune response inducer of Gram-negative bacteria ${ }^{8}$. The close packing of LPS molecules in the outer membrane makes the membrane a strong permeation barrier, which protects cells from the entry of many toxic compounds (including many clinically useful antibiotics). Lipopolysaccharide is composed of hydrophobic lipid A, core polysaccharide, and O-specific polysaccharide (O-antigen) ${ }^{9}$. The lipid A part represents part of the conserved molecular model of LPS and handles most of the biological responses induced by LPS, such as pro-inflammatory responses and septic syndrome ${ }^{10}$. $\mathrm{O}$ antigen helps bacteria evade innate immune responses, including phagocytosis and complement-mediated lysis ${ }^{11}$, 12 . Lipopolysaccharide activates the natural immune system response in the adipose tissue of patients with obesity and type 2 diabetes and increases the risk of endotoxinism and disease ${ }^{5}$.

Patients with type 2 diabetes have significantly increased LPS levels and LPS activity. It positively correlated LPS with inflammation markers and poor blood glucose/lipid control ${ }^{13}$. Late complications (such as massive albuminuria) exacerbate endotoxemia. Clinical reports have shown that the level of LPS in the plasma of patients with severe COVID-19 is increased, and the level of endotoxin is significantly increased ${ }^{14}$. Lipopolysaccharides (LPS) in the blood also induce reactions by interacting with lipopolysaccharide-binding protein (LBP) in the serum ${ }^{15}$. LBP levels of obesity are also high ${ }^{16}$. It closely related serum LBP levels to the hospitalization rate of COVID-19 patients ${ }^{17}$. LBP's plasma levels in hospitalized COVID-19 patients with obvious cardiology are initially high and remain high during hospitalization ${ }^{18}$. It shows that SARS-CoV-2 infection causes an increase in endotoxemia of infected individuals. SARS-CoV-2 infection also induces a "leaky gut" state ${ }^{19}$. SARS-CoV-2 RNA is found in stool samples of hospitalized COVID-19 patients, and it reported diarrhea symptoms ${ }^{20}$. Therefore, the intestinal state caused by endotoxemia is a chronic sequelae of COVID-19.

The core receptors that recognize lipopolysaccharides are CD14, TLR4, and MD-2 ${ }^{21,22}$. The receptor CD14 of lipopolysaccharide LPS exists in two forms: soluble form (sCD14) and membrane-associated (mCD14). Soluble CD14 (sCD14) plays an important role in enhancing the signal response to LPS in cells lacking CD14 on the cell membrane (mCD14) ${ }^{23}$. It increased serum levels of soluble CD14 in asymptomatic patients infected with HIV or lymphadenopathy in normal controls ${ }^{24}$. In SARS-CoV-2 infected patients admitted to the hospital, serum soluble sCD14 levels were also observed presents an increasing state ${ }^{25}$. $\mathrm{mCD} 14$ is regarded as a useful marker molecule for monocytes and macrophages, and it plays an important role in binding the LPS-LBP complex and signal transduction ${ }^{26} . \mathrm{mCD} 14$ binds to lipopolysaccharide and presents it to the TLR4/MD-2 complex, which starts intracellular signaling ${ }^{27}$. The number of cell surface receptor $\mathrm{mCD} 14$ plays a significant role in developing sepsis ${ }^{28}$.

mCD14 is involved in the activation of monocytes induced by lipopolysaccharide(LPS). $\mathrm{mCD} 14$ is a bone marrow monocyte differentiation antigen expressed by monocytes, macrophages, and activated granulocytes ${ }^{29}$. For example, mCD14-positive monocytes increase osteoclast bone resorption by 2-4 times, and osteoclasts are mainly derived from mCD14-positive monocytes ${ }^{30}$. The lipopolysaccharide(LPS) in the blood induces the reaction by interacting with the lipopolysaccharide binding protein (LBP) in the serum. Then the complex stimulates the monocyte surface differentiation antigen $\mathrm{mCD} 14$ protein ${ }^{15}$. Human monocyte $\mathrm{mCD} 14$ is upregulated by lipopolysaccharide ${ }^{31}$. A low LBP concentration enhances LPS-induced monocyte 
activation, while the acute phase increase of LBP concentration inhibits LPS-induced cell stimulation $^{32}$. A decrease in plasma LBP is a surrogate marker for an increase in circulating LPS $^{33}$. In poorly controlled diabetes, $\mathrm{mCD} 14^{+}$monocytes are functionally activated, and markers of myeloid differentiation of monocytes and macrophages are detected ${ }^{34}$.

LBP is a catalyst that catalyzes the binding of LPS to CD14. The N-terminal region is first combined with LPS to form an LPS monomer LBP complex (similar to the substrate enzyme complex), and then the C-terminal region of LBP and CD14 binds to form the LPS/LBP/CD14 complex. LBP escapes from the complex of 3 molecules and enters the next cycle to create the LPS/CD14 complex ${ }^{35}$. One way is to stimulate monocytes, macrophages, and neutrophils through the LPS/LBP complex receptor ${ }^{36}$ (membrane CD14, mCD14) on the cells' membrane to secrete and produce TNF, IL-1, And IL6, and other cytokines, enhancing cell adhesion function and creating inflammation reactions ${ }^{37}$. Another way is soluble CD14 (soluble CD14, sCD14) activates and damages endothelial cells and epithelial cells, and other cells that do not contain mCD14, resulting in a series of biological effects such as inflammation and blood coagulation ${ }^{38}$.

Pro-inflammatory macrophage differentiation is a process that promotes the pathology of a wide range of diseases ${ }^{39}$. Monocytes are circulated innate immune cells and the main participants in cytokine storm and related pathology in COVID-1940. The signal transduction receptor CD14 of LPS is a key marker and a promoter of pro-inflammatory macrophages' function. CD $14^{+} \mathrm{CD} 16^{+}$ inflammatory monocyte subpopulations are found more frequently in COVID-19 patients. $\mathrm{CD} 14^{+} \mathrm{CD} 16^{+}$monocytes also show high levels of IL- 6 expression, which speed up the process of systemic inflammation ${ }^{41,}{ }^{42}$. After being infected with SARS-CoV-2, these people often show hypercytoemia. It indicates that SARS-CoV-2 induces white blood cells to respond to lipopolysaccharide (LPS), and secret cytokines such as tumor necrosis factor- $\alpha$ (TNF-alpha) and interleukin 1 (IL-1 $)^{15}$. Excessive secretion of TNF- $\alpha$ causes endotoxin shock, usually a fatal complication ${ }^{15}$.

After endothelial cells interact with the LPS binding protein and soluble CD14 in plasma, LPS can directly activate the cells. Molecules such as tumor necrosis factor- $\alpha$ and interleukin 1 also activate endothelial cells, providing an indirect activate way for LPS-dependent endothelial cell activation ${ }^{43}$. The vascular endothelium activated by lipopolysaccharides and cytokines observed during sepsis plays an essential role in organ inflammation and blood leukocyte recruitment. The increased $\mathrm{mCD} 14$ intensity expression on circulating monocytes may be vital to the increased inflammatory response observed in patients with the arterial disease ${ }^{44}$. Activated monocytes also show an increased ability to attach to normal endothelial cells, one of the early stages of atherosclerosis ${ }^{45}$. As we all know, arteriosclerosis matters in inducing hypertension. Therefore, if the endotoxin (LPS) of COVID-19 patients cannot be effectively controlled, hypertension may also be induced.

The severity of $\mathrm{HIV}^{46}$ and dengue virus ${ }^{47}$ infections is also related to the level of endotoxin (LPS) in the human body. Lipopolysaccharide activates CD14 $4^{+}$Monocytes. Monocytes are innate immune cells that participate in the complex inflammatory response after migrating to tissues and lymphoid organs, including phagocytosis, antigen presentation, and cytokine secretion ${ }^{48}$. Structural insight reveals that SARS-CoV-2 ORF7a is an immunomodulatory factor of human $\mathrm{CD} 14^{+}$monocytes $^{49}$. The SARS-CoV-2 ORF7a extracellular domain (residues 14-96) binds to $\mathrm{CD}_{14}{ }^{+}$monocytes derived from human peripheral blood mononuclear cell(PBMC) with the highest efficiency, but the binding molecule could not be determined. The combination of ORF7a 
and CD14 monocytes inhibits the antigen presentation ability of these monocytes. SARS-CoV-2 ORF7a triggers $\mathrm{CD} 14^{+}$monocyte antigen presentation inhibition. Orf7a has the ZF domain 'CELYHYQEC'. The ORF7a with the Cys-rich accessory protein may be associated with zinc-binding and interaction of cell antigens (can be activated by a wide range of disulfide) ${ }^{50}$. SARS-CoV-2 ORF7a is an immunomodulatory factor combined with immune cells and triggers a severe inflammatory response. SARS-CoV-2 ORF7a is incubated with CD14 ${ }^{+}$monocytes. In vitro, it starts the reduction of HLA-DR/DP/DQ expression level and promotes cytokines, including IL-6, IL-1 $\beta$, IL-8 and TNF- $\alpha^{51}$. Among hospitalized patients diagnosed with COVID-19, especially in intensive care unit patients, the percentage of peripheral blood $\mathrm{CD} 14^{+} \mathrm{CD} 16^{+}$ inflammatory monocytes is higher ${ }^{52}$. In mild and severe COVID-19 patients, the IL-6 production of inflammation $\mathrm{CD} 14^{+} \mathrm{CD} 16^{+}$monocytes increased significantly, indicating that monocytes are a key factor in the cytokine storm ${ }^{52}$.

The SARS-CoV2 ORF7a protein has a conserved Ig immunoglobulin-like fold, which contains an integrin-binding site, providing a mechanistic hypothesis for the interaction between SARS-CoV2 and the human immune system ${ }^{53}$. The SARS-COV ORF7a expressed in vitro causes apoptosis $^{54}$ through a caspase-dependent pathway $^{55}$, inhibiting cell protein synthesis, and preventing cell cycle progression in $\mathrm{G} 0 / \mathrm{G} 1$ phase $^{56}$, and activating NF-kB ${ }^{57}$. It improves IL-8 promoter activity, activate of $\mathrm{p} 38$ MAP kinase ${ }^{58}$. ORF7a protein is in the endoplasmic reticulum and Golgi apparatus of ORF7a cDNA transfected cells and SARS-CoV1 infected cells ${ }^{59}$. ORF7a may trigger apoptosis by directly interfering with the pro-survival function of Bcl-X. The two proteins show co-localization in the plasma reticulum and mitochondria ${ }^{54}$. Then SARS-CoV-2 ORF7a may reduce the functional mitochondria ${ }^{60}$. SARS-CoV-2 ORF7a and SARS-CoV ORF7a have the same ER retention motif (KRKTE sequence), which indicates that SARS-CoV-2 ORF7a can be incorporated into the viral envelope as structure protein. It has involved ORF7a in immune regulation. But the truncation of the $\mathrm{C}$-terminus negates the anti-immune activity of the protein, which leads to an increased response of type $I$ interferons to viral infections ${ }^{61}$. ORF7a polyubiquitination is mainly formed by K63-linked ubiquitin chains. Then ORF7a can inhibit type I interferon (IFN-I) signaling through STAT2 phosphorylation ${ }^{62}$. In addition, ORF7a of SARS-CoV-2 interacts with ribosomal transporters HEATDR3 and MDN1 ${ }^{63}$, and has been shown to inhibit cell translation in SARS-CoV ${ }^{58}$.

The slight sequence similarity suggests that ORF8 and ORF7a have the same immunoglobulin-like fold, confirmed by structural determination ${ }^{64}$. The SARS-CoV Ig-like virus protein ORF7a interacts with immune cells because of its interaction with the integrin LFA-1 ${ }^{65}$. SARS-CoV2 ORF7a has the critical structural required to bind LFA-1 Cluster and has the associated leukocyte integrin Mac-1 (also known to be expressed by macrophages). The accumulation of ORF7a protein in cells limits the transport of LFA-1/Mac1 integrins to the cell membrane and reduces LFA-1/Mac-1 dependent cell signal transduction, which is an essential cascade of white blood cell function. These include the type II integral membrane protein BST2/tetherin, which is found to prevent virus release and is targeted by the SARS-CoV-2 ORF7a protein to escape immunity ${ }^{66}$.

These different research results indicate ORF7a has a variety of biological activity. So it requires in-depth study of its structure and function. The activity regulation of $\mathrm{CD} 14^{+}$monocytes is usually associated with endotoxin (lipopolysaccharide, LPS). The $\mathrm{ABC}$ transporter-dependent mechanism of lipopolysaccharide biosynthesis is a widespread form ${ }^{67}$. In humans, $14 \mathrm{ABC}$-related 
genes have been reported to be causally related to human diseases, including persistent and hyperinsulinemic hypoglycemia ${ }^{68}$. The SARS-CoV $7 \mathrm{a}$ protein is a type I 122-aa membrane protein $^{59}$. The extracellular domain has a typical 7-strand $\beta$ sandwich fold and a flexible and unstructured region of the immunoglobulin superfamily ${ }^{69}$. The ORF7a protein of SARS-CoV-2 has a similar three-dimensional structure, with residues 80-83 spanning the aa lumen domain ${ }^{70}$. These cavities suggest that ORF7a may have the function of transmembrane molecular transport. Therefore, we believe that ORF7a may have the function of ABC-transporter.

This study adopted motif search, and molecular docking methods to study the SARS-CoV-2 virus protein. The results showed ORF7a had the domains of ABC transporter: ATP binding domain and transmembrane domain. The $\mathrm{ABC}$ transport domain of ORF7a transported molecules such as metal ions, heme, and had a multi-drug efflux function. ORF7a also has lipopolysaccharide synthesized domains. The extracellular ORF7a with the N-terminus and C-terminus cut off still has the BPI11/LBP domain (CELYHYQECVR) and two ATP-binding motifs ("HYQEC" and "TYEGNS"). Extracellular ORF7a can bind to CD14 molecules like LBP.

\section{Data sets and methods}

\subsection{Data set}

1. The sequences of SARS-COV-2 protein. The SARS-COV-2 protein ORF7a.

2. The sequences of bacterial ABC-related and lipopolysaccharide-related proteins. We downloaded the sequences of the 688,680 bacterial ABC-related proteins and 473,607 lipopolysaccharide-related proteins from the UniProt dataset. The search keywords are "Bacterial $+\mathrm{ABC}+$ transporter" and "Lipopolysaccharide", respectively.

3. Crystal structures of extracellular ORF7a. we downloaded extracellular ORF7 (PDB ID: 7ci3) from the PDB database.

4. Crystal structures of CD14. Human CD14 molecules (PDB ID: 4glp) and Mus musculus CD14 molecules (PDB ID: 1WWL)

\subsection{Localized MEME tool to scan for conserved domains.}

The analysis steps are listed as follows (for example "Bacterial+ABC+transporter") :

1. Download MEME from the official website and subsequently install in the virtual machine ubuntu operating system. The virtual machine was VM 15.2.

2. Download the sequences of SARS-COV-2 protein from NCBI official website.

3. Download the fasta format sequence of $A B C$-related protein from Uniprot official website, respectively. The search keyword was "Bacterial+ABC+transporter".

4. For each sequence in all ABC-related proteins, paired with each SARS-COV-2 protein sequence to generate fasta format files for MEME analysis.

5. For the files generated in Step 4, a batch of 50000 was used to create several batches. It was considered as the limited space of the virtual ubuntu system.

6. In ubuntu, searched the conserved domains (E-value $<=0.05$ ) of SARS-COV-2 protein and $\mathrm{ABC}$-related protein with MEME tools in batches.

7. Collected the result files of conserved domains. Find the domain name corresponding to the motif from the uniprot database.

8. We analyzed the domains' activity of the each SARS-COV-2 protein according to the 
characteristics of the $\mathrm{ABC}$-related protein domains.

\subsection{Docking of Discovery Studio 2016}

We used Discovery-Studio's protein docking (ZDOCK) to determine whether ORF7a and CD14 were bound.

\section{Results}

\subsection{ABC transport system of ORF7a protein}

$\mathrm{ABC}$ transporter is first discovered in bacteria. It is a transport ATPase (transport ATPase) on the bacterial plasma membrane and belongs to a large and diverse protein family. The $A B C$ transporter contains at least two conserved regions: a highly conserved ATP binding cassette (ABC) and a less conserved transmembrane domain (TMD). In some bacterial transport proteins, these regions are on different polypeptides. Most $\mathrm{ABC}$ transporters act as dimers and consist of four domains: two $\mathrm{ABC}$ modules and two TMDs. $\mathrm{ABC}$ transporters usually comprise multiple subunits, one or two of which are transmembrane proteins, and one or two are membrane-associated AAA ATPase. The function of the intact inner membrane protein is to transport the substrate across the membrane and to recognize the substrate ${ }^{71,72}$. The ATPase subunit binds the adenosine triphosphate (ATP) and hydrolysis ATP to provide the energy required for substrate transport across the membrane to absorb or export the substrate.

$\mathrm{ABC}$ transporter ( $\mathrm{ABC}$ transporter) was first discovered in bacteria. It is a transport ATPase (transport ATPase) on the bacterial plasma membrane. It belongs to a large and diverse protein family. The $\mathrm{ABC}$ transporter contains at least two conserved regions: a highly conserved ATP binding cassette $(\mathrm{ABC})$ and a less conserved transmembrane domain (TMD). In some bacterial transport proteins, these regions are located on different polypeptides. Most $\mathrm{ABC}$ transporters act as dimers and consist of four domains, two $\mathrm{ABC}$ modules, and two TMDs. $\mathrm{ABC}$ transporters usually consist of multiple subunits, one or two of which are transmembrane proteins. One or two are membrane-associated AAA ATPase. The function of the intact inner membrane protein is to transport the substrate across the membrane and to recognize the substrate $(64,65)$. ABC transporter dimerizes by binding ATP. ATP is hydrolyzed and depolymerized. The substrate-bound to it is transferred to the other side of the membrane through conformational changes. The ATPase subunit uses the energy of adenosine triphosphate (ATP) binding and hydrolysis to provide the energy required for substrate transport across the membrane to absorb or export the substrate.

We downloaded $\mathrm{ABC}$ protein-related sequences from the Uniprot database with the keyword "Bacterial+ABC+transporter". Then used the local MEME tool to search for the conserved domains of these proteins and ORF7a protein. In the initial search results, there were too many motif fragments for one domain. For the convenience of analysis, we had merged the motif sequences by domain. The search results of domains and motifs related to the $\mathrm{ABC}$ transport system are shown in Table 1. ORF7a has ATP binding domains (AAA, AAA_15, CBIO, CBS, OppC_N, STAS, TOBE_2) and transmembrane domains (ABC transmembrane type-1, ABC transporter, ABC_tran_Xtn, ABC_transp_aux, ABC2_membrane). AMP-binding (Interpro entry: IPR000873) is the AMP binding region ${ }^{73}$. The ATP binding domain overlaps the transmembrane domain."MKIILFLALITLATCELYHYQECVRGTTVLLKEPCSSGTYEGNSPFHPLADNKFAL TCFSTQFAFACPDGVKHVYQLRARSVSPKLFIRQEEVQELYSPIFLIVAAIVFI” (residues 1 - 
110) motif has the function of ATP binding and $\mathrm{ABC}$ transmembrane transport.

ATP binding sites (ABC). The ATP binding sites are in AAA, AAA_15, CBIO, CBS, OppC_N, STAS, TOBE_2 domains. AAA (Pfam: PF13173) is an ATPase ${ }^{74}$ related to a variety of cellular activities, an ATP-dependent protein clamp that can achieve ATP-dependent proteasome function and ATP-dependent anchoring of proteins. AAA (ATPases Associated with diverse cellular Activities) protein couples the chemical energy provided by ATP hydrolysis with conformational changes and converts it into mechanical force exerted on macromolecular substrates $^{75}$. The AAA protein of the cyclic P-ring NTPase exerts its activity through energy-dependent remodeling or translocation of macromolecules ${ }^{76,77}$. It involved AAA protein in protein degradation and membrane fusion. The AAA domain contains two sub-domains, an $\mathrm{N}$-terminal alpha/beta domain that binds and hydrolyzes nucleotides (Rossmann fold) and a C-terminal alpha-helix domain ${ }^{78}$. AAA_15 (Interpro entry: IPR041685) is the AAA ATPase domain, which contains a P-ring motif characteristic of the AAA superfamily ${ }^{79}$. CBIO (Interpro entry: IPR005876) is the cobalt transporter ATP binding subunit ${ }^{80}$. CBS domain (Interpro) entry: IPR000644) binds to ligands with adenosine groups ${ }^{81}$, such as AMP, ATP, and S-AdoMet. OppC_N (Interpro entry: IPR025966) is an oligopeptide transport permease C-like, N-terminal domain, and is a highly conserved ATP binding cassette transporter ${ }^{82}$. The STAS (sulfate transporter and AntiSigma factor antagonist) domain (Interpro entry: IPR002645) was found in the C-terminal region of the bacterial anti-sigma factor antagonist (ASA) and SLC26 (SulP) anion transporter. The STAS domain of ASA SpoIIAA binds GTP and ATP ${ }^{83}$, and has weak NTPase activity. The decisive sequence conservation shows that the STAS domain may have general NTP binding activity. TOBE_2 (Interpro entry: IPR013611) is a transport-related OB, type 2, found in the ABC transporter after the ATPase domain ${ }^{84}$. In summary, we can see that there are two ATP binding motifs in Table 1, "HYQEC" (residues 19-23) and "TYEGNS" (residues 39-44), the former is at the N-terminal, and the latter is at at the middle near C-terminal. The sites of AAA_15, CBIO, CBS, OppC_N, STAS, TOBE_2 domains are ATP binding regions. CBIO and TOBE_2 have two ATP binding sites at the $\mathrm{N}$ and middle, respectively. AAA_15 and CBS have a $\mathrm{N}$-terminal ATP binding site, and OppC_N and STAS have a middle ATP binding site. AAA spans the ATP binding site and the TM binding site.

Transmembrane structure binding sites (TMD). The transmembrane structure binding sites include $\mathrm{ABC}$ transmembrane type-1, $\mathrm{ABC}$ transporter, $\mathrm{ABC}$ _tran_Xtn, $\mathrm{ABC}$ _transp_aux, ABC2_membrane domains. ABC transmembrane type-1 (Interpro entry: IPR011527) represents the transmembrane domain when the TMD and $\mathrm{ABC}$ regions are found in the same protein ${ }^{85}$. ABC_transp_aux (Interpro entry: IPR019196, Pfam: PF09822) belongs to the ABC type non-characteristic transport system. This domain is found in various eukaryotic and prokaryotic intraflagellar transporters and several hypothetical proteins involved in a gliding motion. ABC2_membrane (Pfam: PF01061, Interpro entry: IPR013525) belongs to the transmembrane structure of the ABC-2 transporter ${ }^{86}$. ABC transporter (Pfam: PF00005, Interpro entry: IPR003439) belongs to the ATP-binding cassette (ABC) superfamily, which uses the hydrolysis of ATP to provide energy for various biological systems ${ }^{87}$. The $A B C$ transporter comprises at least two conserved regions: the highly conserved ATP-binding cassette (ABC) and the less conserved transmembrane domain (TMD). ABC_tran_Xtn (Interpro entry: IPR032781) is the $\mathrm{ABC}$-transporter extension domain bound to the ribosome to stabilize the interaction ${ }^{88}$. In summary, the $\mathrm{ABC}$ transmembrane domain (TMD) of ORF7a in Table 1 is at sites 1-110, and the 
first half of the sequence overlaps with the two ATP binding sites.

The type of ORF7a transport substance. We continued to sort out the "Bacterial + ABC + transporter" domain's search results, then summarized the ORF7a transportable substances and the corresponding motifs (Table 2). Table 2 shows that ORF7a can transport metal ions (molybdenum, cobalt), heme, purine nucleoside/riboflavin, amino acids (branched-chain amino acids Leu/Ile/Val, methionine), oligopeptides, sugars(D-galactose, lactose), ribose, vitamins (Vb1/Vb2), periplasmic solutes, salts (citrate, phosphate, C4-dicarboxylate, molybdenum sulfate), alkali (glycine betaine), and other molecules. It has also multi-drug efflux function. The sites that achieve the transport function are in the $\mathrm{ABC}$ transporter domain (residues 1-110).

The domains involved in transport are: Bmp domain (Interpro entry: IPR003760, PFAM: PF02608) is a basic membrane lipoprotein and also a substrate-binding protein of ABC transporter. Examples include PnrA (also known as TmpC or TP0319) and RfuA (also known as Tpn38 or TP0298) from Treponema pallidum. PnrA transports purine nucleosides ${ }^{89}$, while RfuA transports riboflavin ${ }^{90}$. CBIO (Interpro entry: IPR005876) is the cobalt transporter ATP binding subunit ${ }^{80}$. CitMHS domain (Interpro entry: PF16980) is citric acid salt transporter family belongs to the NhaD-like permease superfamily of $\mathrm{Na}+/ \mathrm{H}+$ antiporters. The DctM domain (Interpro entry: IPR010656) is the DctM subunit of the TRAP C4-dicarboxylate transport system permease. The C4-dicarboxylate transport system can absorb C4-dicarboxylates such as succinate, fumarate, and malic acid ${ }^{91}$. DctM is a complete membrane protein and one component of the periplasmic TRAP vector $^{92}$. DUF3382 (PFAM: PF02653) is a high-affinity permease for the branched-chain amino acid transport system ${ }^{93}$. DUF3708 (PFAM: PF00528, Interpro entry: IPR035906) is the D-methionine transport system permease protein MetI, the helix subset, observed in the larger transmembrane subunits of the molybdate (ModBC) and maltose (MalFGK) ABC transporters ${ }^{94}$. FtsX (Interpro entry: IPR003838, PFAM: PF02687) is an ABC3 transporter permease protein domain. It is a heme transport system. The permease protein HrtB may be part of the ABC transporter complex involved in the import of $\mathrm{Hemin}^{95}$. It is also the transmembrane protein LolC of the lipoprotein release system ${ }^{96}$. FtsX_ECD (Interpro entry: IPR040690) is the extracellular domain (ECD) found in FtsX, which is a homolog of transmembrane PG hydrolase modulator ${ }^{97}$. HMA (Interpro entry:IPR006121) is a conserved domain found in many heavy metal transport or detoxification proteins ${ }^{98}$. MacB_PCD (Interpro entry: IPR025857) is a periplasmic core domain found in various $A B C$ transporters, similar to AcrB multidrug efflux transporters ${ }^{99}$. MatC_N (Interpro entry: IPR009827) is the N-terminal region of the dicarboxylate carrier protein MatC ${ }^{100}$. MatC protein is a complete membrane protein that can be used as a malonate carrier. MFS (Interpro entry: IPR011701) targets a wide spectrum of substrates, including ions, carbohydrates ${ }^{101}$, lipids, amino acids and peptides, nucleosides and other small molecules in both directions across the membrane ${ }^{102}$. The NIL domain (Interpro entry: IPR018449) is at the C-terminus of the ABC transporter involved in D-methionine transport and many ferredoxin-like proteins. The NMT1/THI5 series (Interpro entry: IPR027939) are the substrate-binding components of the ABC transporters of vitamins B1 and B2 ${ }^{103}$. OppC_N domain (Interpro entry: IPR025966) is the $\mathrm{N}$-terminal domain of oligopeptide transport permease C-like ${ }^{82}$. OpuAC (Interpro entry: IPR007210, PFAM: PF04069) is the ABC-type glycine betaine transport system ${ }^{104}$. $\mathrm{PBPb}$ (Interpro entry: IPR001638, SMART: SM00062) is the 3/N-terminal domain of the MltF solute-binding protein family ${ }^{105}$. The PDGLE domain (Interpro entry: IPR025937) was also found in the energy coupling factor transporter substrate capture protein NikMN, indicating that it may 
play a role in metal transport. Peripla_BP_4 (Interpro entry: IPR025997, PFAM: PF13407) participates in the active transport of galactose ${ }^{106}, \mathrm{AI}-2^{107}$, and acidic amino acids ${ }^{108}$ across the plasma membrane of the solute. Peripla_BP_6 (Interpro entry: IPR028081, PFAM: PF13458) is a leucine binding protein and Leu / Ile/Val binding protein, the main receptor of the leucine transport system ${ }^{109}$. SBP_bac_5 (Interpro entry: IPR000914) is involved in the active transport of solutes across the plasma membrane ${ }^{110}$. The SSD domain (Interpro entry: IPR000731) is a sterol sensing domain ${ }^{111}$. The STAS domain (Interpro entry: IPR002645) is found in the C-terminal region of the bacterial anti-sigma factor antagonist (ASA) and SLC26 (sulfate) anion transporter ${ }^{83}$. ABC_transpr_TagH (Interpro entry: IPR015860) participates in the export of teichoic acid ${ }^{112}$. The TOBE_2 domain (Interpro entry: IPR013611, PFAM: PF08402) is related to the transport of molybdenum (SWISSPROT: P46930) and sulfate (SWISSPROT: P16676) ${ }^{84}$.

Lipoprotein release domains. We searched again from the above $\mathrm{ABC}$ domain results to find the lipoprotein release domains. The SBP_bac_5 domain (residues 10-100) has lipoprotein activity $^{105}$. Bmp (residues 15-25, 43-48, 51-82) is the membrane lipoprotein region ${ }^{113}$. Peripla_BP_4 (residues 14-78) and PBPb (residues 14-59) have membrane-anchored lipoprotein activity ${ }^{105}$. FtsX (residues 10-24, 34-90) is the release position of lipoprotein ${ }^{114}$.

\subsection{ORF7a protein could synthesize lipopolysaccharide}

The translocation of LPS from the inner membrane IM to the outer membrane OM is unidirectional ${ }^{115}$. All components of LPS (lipid A, core, and $\mathrm{O}$ antigen) are synthesized on the internal lobules of IM, where lipid A and core are connected to form the so-called rough LPS. The rough LPS and O antigens pass through the IM independently and are ligated on the lobules of the IM 9 . Then LPS is extracted from IM by ABC transporter.

We downloaded the protein sequence related to lipopolysaccharide from the UniProt database. Then used the local MEME tool to compare lipopolysaccharide-related proteins with SARS-COV-2 protein ORF7a to find the conserved domains. We combined the motifs according to the structural domains of the comparison results (Table 3). Table 3 shows that ORF7a can synthesize Lipids, Core polysaccharide and O-specific polysaccharide and assemble the three molecules into lipopolysaccharide. The sites involved in lipopolysaccharide synthesis are 1-120: "MKIILFLALITLATCELYHYQECVRGTTVLLKEPCSSGTYEGNSPFHPLADNKFALTCFSTQ FAFACPDGVKHVYQLRARSVSPKLFIRQEEVQELYSPIFLIVAAIVFITLCFTLKRKT".

The relevant domains of synthetic lipopolysaccharide are: Acyl_transf_3 domain (Interpro entry: IPR002656) is the acyltransferase 3 domain. Acyl_transf_3 includes NolL protein, and NolL protein functions as an acetyltransferase ${ }^{116}$. Acetyltransferase is an important enzyme in the process of lipid synthesis. The CTP_transf_like domain (Interpro entry: IPR004821) is a cytidine transferase-like domain. This protein family includes lipopolysaccharide core biosynthesis protein $\mathrm{KdtB}^{116}$. The Glyco_transf_8C domain (Interpro entry: IPR013645) is at the C-terminus of the glucosyltransferase and galactosyltransferase proteins. Glyco_transf_8C has lipopolysaccharide 3 - $\alpha$-galactosyltransferase activity, jointing the process of lipopolysaccharide biosynthesis. The Glycos_transf_1 domain (Interpro entry: IPR001296) is family 1 of glycosyltransferases. Glycos_transf_1 domain protein transfer UDP, ADP, GDP or CMP linked sugars to various substrates, including glycogen, fructose 6-phosphate, and lipopolysaccharide. Glycos_transf_1 can take part in various biosynthetic processes, including exopolysaccharide biosynthesis and lipopolysaccharide core biosynthesis ${ }^{117}$. PglL_A domain (Interpro entry: IPR031726) is a protein glycosylation ligase domain. PglL O-oligosaccharyltransferase differs from WaaL O-antigen 
ligases. PglL O-oligosaccharyl transferase (O-OTase) transfers oligosaccharides to serine or threonine in proteins ${ }^{118}$. Glycos_transf_N domain (Interpro entry: IPR007507) is the N-terminus of 3-deoxy-D-manno-octulosonic-acid transferase. Glycos_transf_N can transfer activated sugars to various substrates, including glycogen, fructose 6-phosphate, and lipopolysaccharide ${ }^{119}$. Glycos_transf_N proteins with this domain transfer UDP, ADP, GDP, or CMP-linked sugars. A signal peptide flanks the N-terminus of this region. Rubredoxin_2 domain (Interpro entry: IPR041166) is LapB (lipopolysaccharide assembly protein B) ${ }^{120}$, rubredoxin metal-binding domain. The rubredoxin-like domain (Interpro entry: IPR024934) is a similar rubredoxin domain. The Wzz domain (Interpro entry: IPR003856, PFAM: PF02706) is the N-terminal domain of the polysaccharide chain length determinant. The chain length determining protein (or wzz protein) is involved in lipopolysaccharide (lps) biosynthesis, giving the O-antigen component of lps a modal distribution of chain length ${ }^{121}$.

\subsection{Extracellular ORF7a could bind to the CD14 antigen molecule}

Structural insight reveals that SARS-CoV-2 ORF7a is an immunomodulatory factor of human $\mathrm{CD} 14^{+}$monocytes ${ }^{49}$. SARS-CoV-2 ORF7a is a type I transmembrane protein with 121 amino acid. Consists of the N-terminal signal region (residues 1-15), Ig-like extracellular domain (residues 16-96), hydrophobic transmembrane domain (residues 97-116), and typical endoplasmic reticulum retention motifs (residues 117-121). SARS-CoV-2 ORF7a has a conserved Ig-like $\beta$-sandwich fold of seven $\beta$ chains. The SARS-CoV-2 ORF7a extracellular domain protein (residues 14-96) binds to $\mathrm{CD} 14^{+}$monocytes from human peripheral blood mononuclear cells (PBMC) with the highest efficiency. However, the interaction pattern between SARS-CoV-2 ORF7a and white blood cells differs from the ICAM3-LFA-1 complex ${ }^{49}$.

In the synthesis domain analysis of lipopolysaccharide, we found that ORF7a had the BPI1 domain (CELYHYQECVR, 15-25). BPI1 domain (Interpro entry: IPR017942, SMART: SM00328) represents the N-terminal domain found in several lipid-binding serum glycoproteins. It includes bactericidal permeability-increasing protein (BPI), lipopolysaccharide-binding protein (LBP). BPI can bind to and neutralize lipopolysaccharides in the outer membrane ${ }^{122}$. Lipopolysaccharide binding protein (LBP) is an endotoxin binding protein closely related to BPI and acts synergistically to promote the host's comprehensive response to invasion ${ }^{123}$. ORF7a (residues 14-96) without the N-terminal signal peptide and the transmembrane region still has the BPI /LBP domain and two ATP binding sites. The BPI1/LBP domain is at the N-terminus of the extracellular ORF7a and is also at the first $\beta$-sandwich fold of the Ig-like fold of the extracellular domain (Figure 1.A). ORF7a has two ATP binding sites, "HYQEC" and "TYEGNS", the former is in the N-terminal BPI1/LBP domain, and the latter is behind the relative direction of the BPI/LBP domain. Interestingly, the ABC transporter domain of ORF7a includes the N-terminal signal region and the transmembrane region. The BPI1 domain of ORF7a is also in the vital region of $\mathrm{ABC}$ transport and lipopolysaccharide synthesis domain and is also the lipopolysaccharide-binding region. So the BPI1 domain of extracellular ORF7a is also a lipopolysaccharide-binding domain. It indicates that the splicing of the $\mathrm{N}$-terminal signal peptide and the transmembrane region may trigger ORF7a to lose its $\mathrm{ABC}$ transport function and release itself outside the infected cell.

In order to determine whether the extracellular ORF7a binds to the CD14 molecule, we made the extracellular ORF7a to the human CD14 molecule for protein docking. The PDB database only has human CD14 molecules (PDB ID: 4glp) and Mus musculus CD14 molecules (PDB ID: 
1WWL). The human CD14 molecule (4glp) is a monomer, and the Mus musculus CD14 molecule $(1 \mathrm{WWL})$ is a dimer. The C-terminal sequence of the CD14 molecule is mainly involved in GPI anchoring ${ }^{124}$. C-terminal truncation does not affect the relative biological activity of LPS binding ${ }^{125}$. The relevant biologically active site of LPS is within the N-terminal half of the CD14 protein $^{126}$. Our protein docking results show that the end of the N-terminal of extracellular ORF7a can bind to the 1WWL dimer, but the extracellular ORF7a cannot bind to 4glp.

Considering that the CD14 sequence of the PDB database had a truncation problem, we used the Robetta server to model the structure of the human CD14 molecule and then adopted the extracellular ORF7a and the modeled human CD14 for protein docking (Figure 1.B). Figure 1.B shows that the extracellular ORF7a can bind to the human CD14. The extracellular ORF7a binding sites are in the middle and C-terminal beta folding region. The one binding area of CD14 is near the alpha-helix between the second beta-sheet and the third beta-sheet. Another binding area is closed to alpha-helix between the third beta-sheet and the fourth beta-sheet. The N-terminal alpha-helix of CD14 is behind the binding region, which shows that the N-terminal structure of CD14 plays a vital role in assisting the binding of extracellular ORF7a to CD14. The N-terminal BPI1/LBP domain of extracellular ORF7a is outside. The first ATP binding site("HYQEC") is in the BPI1/LBP domain. The second ATP binding site ("TYEGNS") is close to the N-terminal active pocket of CD14.

The above domain search and protein docking results show that the N-terminal BPI1/LBP domain of extracellular ORF7a has functioned as binding lipopolysaccharide, the C-terminal and middle part of extracellular ORF7a bind CD14. Therefore, we believed extracellular ORF7a had similar LBP activity and could help to form the LPS-ORF7a-CD14 complex, then ORF7a fell off and promoted the binding of LPS and CD14. However, the N-terminal of extracellular ORF7a is outside the N-terminal active pocket of CD14. We speculated that the two ATP-supplying energy structures promoted the rotation of the extracellular ORF7a around the two ATP sites' central axis and accurately delivered the N-terminal LPS into the N-terminal active pocket of CD14.

\section{Discussion}

\subsection{Lipopolysaccharide made SARS-COV-2 virus be more aggressive.}

SARS-CoV-2 ORF7a and SARS-CoV ORF7a share the same ER retention motif (C-terminal KRKTE sequence), suggesting that SARS-CoV-2 ORF7a may be incorporated into the viral envelope as SARS-CoV ORF7 $\mathrm{a}^{49}$. Viruses bind to bacteria or directly bind to free Lipopolysaccharide(LPS), enhancing their attachment to the ACE2 receptor on the host cell surface. This interaction significantly increases viral infectivity and promotes hypercytokineemia

${ }^{127}$. Therefore, LPS makes the virus spread more efficiently. In addition, SARS-COV-2 virus protein synthesized and transported LPS, which activated monocytes or phagocytes through CD14 receptors. When LPS increased, the number of activated phagocytes increased significantly. The swallowed behavior for red blood cell and iron by activated phagocytes was enhanced. There were more heme and iron in phagocytes. ROS damage theory ${ }^{128}$ believes that the SARS-COV-2 virus parasitizes in the vesicles or lysosomes of phagocytes. After the virus binds to the heme in the vesicles or lysosomes, it is more aggressive. It attacks the phagocytes' lysosome membrane by generating ROS. The lysosomal membrane ruptures after being damaged by ROS to release hydrolase. The phagocytes will die and rupture due to autophagy reaction and released virus 
particles. The nearby phagocytes swallows the virus particles again and continues a vicious circle. The surrounding tissues will therefore produce a robust inflammatory response and fibrosis.

\subsection{ORF7a enhanced the ability of infected cells to resist $T$ cells, NK cells, and drugs}

Microorganisms often use cell surface polysaccharides to build expanded barriers to prevent their host's defense mechanisms ${ }^{129}$. In this study, it was found that ORF7a can synthesize lipopolysaccharide (LPS). After a lot of LPS bound to the infected cell's surface, LPS was equivalent to the infected cell's shield, making the SARS-COV-2 infected cell's outer membrane like a "cell wall". Infected cells significantly reduced cytotoxic T cells and NK cells' ability to destroy the infected cell membrane (such as perforation).

Simultaneously, the transport function of the $\mathrm{ABC}$ transporter for drugs increased bacteria resistance and made antibiotics and other drugs ineffective. At present, COVID-19 patients in some areas had a dual infection of fungus and bacteria in their lungs. Long-term antibiotics for these patients increased bacterial resistance. For cells infected with the SARS-COV-2 virus, similar drug resistance problems would also arise because of the $\mathrm{ABC}$ transport function of ORF7a protein. Therefore, some researchers speculated from the statistical data that sickle cell infected SARS-COV-2 showed resistance but are very confused about it.

\subsection{Mutations in the extracellular domain of ORF7a enhanced its LBP-like activity}

The sequence alignment of SARS-CoV-2 ORF7a and SARS-CoV ORF7a shows that SARS-CoV-2 ORF7a has a conserved Ig-like $\beta$ sandwich fold with seven $\beta$ chains. Most of the sequence variation is distributed in the extracellular domain (8-residue variation), so the potential protein function is diversified. Interestingly, this part represents the internal disordered C-terminal region of the SARS-CoV ORF7a protein, which the 3D structure of the ORF7a protein of SARS implied ${ }^{130}$. Western blot analysis with a specific anti-ORF7a antibody indicates that SARS-CoV ORF7a is a new structural protein in viral particles ${ }^{131}$. The deletion of nucleotides at specific sites leads to the fusion of ORF7a and ORF7b ${ }^{132}$. The rare Greek SARS-CoV-2 variant contains a premature stop codon in ORF7a, causing the protein to be truncated. Premature termination will abolish the 28 amino acids at the C-terminus of the ORF7a protein ${ }^{69}$. This mutation may represent the continued adaptation of the virus to human cells ${ }^{133}$. The C-terminal truncation of ORF7a will undoubtedly produce sub-proteins with LBP activity. The extracellular domain mutation makes us worried that there may be short ORF7a that enhances LBP-like activity.

\section{Conclusion}

Diabetes and obesity are susceptible to COVID-19 disease and are also essential factors that lead to the worsening of COVID-19 condition. Because of COVID19 pneumonia, the blood sugar control of diabetic patients may deteriorate, which may be insulin resistance that can induce the accumulation of lipopolysaccharide (LPS)-dependent macrophages in white adipose tissue (WAT). Structural analysis studies have shown that SARS-CoV-2 ORF7a is an immunomodulatory factor for human CD14 + monocytes and phagocytes. It usually relates the regulation of CD14 + monocytes to endotoxin (lipopolysaccharide, LPS). The ABC transporter-dependent lipopolysaccharide biosynthesis mechanism is a common form. Therefore, it is of great 
significance to study the pathological mechanism of the relationship between SARS-CoV-2 virus and lipopolysaccharide, $\mathrm{ABC}$ transport, and diabetes.

In this study, bioinformatics techniques such as domain search, molecular docking were used to study the relationship between SARS-COV-2 virus ORF7a protein and lipopolysaccharide. The results showed that the transmembrane protein ORF7a protein had ABC transporter domains: ATP binding and $\mathrm{ABC}$ transmembrane domains. The $\mathrm{ABC}$ transporter domain of ORF7a transported molecules such as metal ions, heme, nucleosides, amino acids and oligopeptides, sugars, vitamins, salts and alkalis, and it had a multi-drug efflux function. ORF7a had domains for synthetic lipopolysaccharide. The lipopolysaccharide synthesized by ORF7a bound to CD14 molecules through LBP protein to activate CD14 $4^{+}$monocytes. The extracellular ORF7a with the $\mathrm{N}$-terminus and C-terminus cut off still had the BPI1 domain (CELYHYQECVR) and two ATP-binding motifs. The first ATP binding site ("HYQEC") is in the BPI1/LBP domain. The second ATP binding site ("TYEGNS") is behind the relative direction of the BPI/LBP domain. Extracellular ORF7a had lipopolysaccharide - binding protein (LBP) - like activity, could be combined with CD14 antigen molecules.

We speculated that the lipopolysaccharide synthesized by the SARS-COV-2 virus protein made the SARS-COV-2 virus more effectively attached to the ACE2 receptor on the host cell surface. The interaction significantly increases viral infectivity and promotes hypercytokineemia. More lipopolysaccharides activated monocytes and phagocytes to release many inflammatory factors, destroyed adipose tissue and vascular endothelial tissue, and induced diabetes and high blood pressure. Cells infected by the SARS-COV-2 virus often used cell surface polysaccharides to build expanded barriers to prevent their host's defense mechanisms. Lipopolysaccharide enhanced the ability of infected cells to resist $\mathrm{T}$ cells, NK cells, and drugs. For cells infected with the SARS-COV-2 virus, similar drug resistance problems would also arise because of the ABC transport function of ORF7a protein.

\section{Declarations}

\section{Ethics approval and consent to participate}

Not applicable.

\section{Consent for publication}

Not applicable.

\section{Availability of data and material}

The datasets and results supporting the conclusions of this article are available at https://pan.baidu.com/s/1IKycgDL-xAThbIuaoOE3Rw , code: ug00. Or: https://mega.nz/folder/MmpVCCgL\#akh3TRzW83vfMeSSg2SFHg

\section{Competing interests}

The authors declare that they have no competing interests.

\section{Funding}

This work was funded by a grant from the Talent Introduction Project of Sichuan University of Science and Engineering (award number: 2018RCL20, grant recipient: WZL). 


\section{Author's contribution}

Funding was obtained by WZL. Besides, design, analysis and writing are finished by WZL, while data curation and manuscript check are undertaken by HLL. Both authors have read and agreed to the published version of the manuscript.

\section{Acknowledgements}

Not applicable.

\section{Author details}

${ }^{1}$ School of Computer Science and Engineering, Sichuan University of Science \& Engineering, Zigong, 643002, China.

${ }^{2}$ School of Life Science and Food Engineering, Yibin University, Yibin, 644000, China.

\section{References}

1. Caussy C, Pattou F, Wallet F, et al. Prevalence of obesity among adult inpatients with COVID-19 in France. Lancet Diabetes Endocrinol 2020; 8(7): 562-4.

2. Kruglikov IL, Shah M, Scherer PE. Obesity and diabetes as comorbidities for COVID-19: Underlying mechanisms and the role of viral-bacterial interactions. Elife 2020; 9: e61330.

3. Kruglikov IL, Scherer PE. The role of adipocytes and adipocyte-like cells in the severity of COVID-19 infections. Obesity 2020; 28(7): 1187-90.

4. Lim S, Bae JH, Kwon H-S, Nauck MA. COVID-19 and diabetes mellitus: from pathophysiology to clinical management. Nature Reviews Endocrinology 2020: 1-20.

5. Creely SJ, McTernan PG, Kusminski CM, et al. Lipopolysaccharide activates an innate immune system response in human adipose tissue in obesity and type 2 diabetes. American Journal of Physiology-Endocrinology and Metabolism 2007; 292(3): E740-E7.

6. Belančić A. Gut microbiome dysbiosis and endotoxemia-Additional pathophysiological explanation for increased COVID-19 severity in obesity. Obesity Medicine 2020; 20: 100302

7. Caceres AI, Kaelberer MM, Jordt S-E. Late Breaking Abstract-The airway sensory nerve transcriptome: evidence for neuropathic injury following lipopolysaccharide induced lung inflammation. Eur Respiratory Soc; 2020.

8. Ulevitch R, Tobias P. Receptor-dependent mechanisms of cell stimulation by bacterial endotoxin. Annual review of immunology 1995; 13(1): 437-57.

9. Raetz CR, Whitfield C. Lipopolysaccharide endotoxins. Annual review of biochemistry 2002; 71(1): 635-700.

10. Cohen J. The immunopathogenesis of sepsis. Nature 2002; 420(6917): 885-91.

11. Goebel EM, Wolfe DN, Elder K, Stibitz S, Harvill ET. O antigen protects Bordetella parapertussis from complement. Infection and immunity 2008; 76(4): 1774-80.

12. Skurnik M, Bengoechea JA. The biosynthesis and biological role of lipopolysaccharide O-antigens of pathogenic Yersiniae. Carbohydrate research 2003; 338(23): 2521-9.

13. Jayashree B, Bibin Y, Prabhu D, et al. Increased circulatory levels of lipopolysaccharide (LPS) and zonulin signify novel biomarkers of proinflammation in patients with type 2 diabetes. Molecular and cellular biochemistry 2014; 388(1): 203-10.

14. Sirivongrangson P, Kulvichit W, Payungporn S, et al. Endotoxemia and circulating bacteriome in severe COVID-19 patients. Intensive care medicine experimental 2020; 8(1): 1-15. 
15. Wright SD, Ramos RA, Tobias PS, Ulevitch RJ, Mathison JC. CD14, a receptor for complexes of lipopolysaccharide (LPS) and LPS binding protein. Science 1990; 249(4975): 1431-3.

16. Sun L, Yu Z, Ye X, et al. A marker of endotoxemia is associated with obesity and related metabolic disorders in apparently healthy Chinese. Diabetes Care 2010; 33(9): 1925-32.

17. Zhou S, Butler-Laporte G, Nakanishi T, et al. Circulating Proteins Influencing COVID-19 Susceptibility and Severity: a Mendelian Randomization Study. medRxiv 2020.

18. Hoel H, Heggelund L, Reikvam DH, et al. Elevated markers of gut leakage and inflammasome activation in COVID-19 patients with cardiac involvement. Journal of internal medicine 2020.

19. Kruglikov IL, Scherer PE. Preexisting and inducible endotoxemia as crucial contributors to the severity of COVID-19 outcomes. PLoS Pathogens 2021; 17(2): e1009306.

20. Xiao F, Tang M, Zheng X, Liu Y, Li X, Shan H. Evidence for gastrointestinal infection of SARS-CoV-2. Gastroenterology 2020; 158(6): 1831-3. e3.

21. Kim J-I, Lee CJ, Jin MS, et al. Crystal structure of CD14 and its implications for lipopolysaccharide signaling. Journal of Biological Chemistry 2005; 280(12): 11347-51.

22. Fujihara M, Muroi M, Tanamoto K-i, Suzuki T, Azuma H, Ikeda H. Molecular mechanisms of macrophage activation and deactivation by lipopolysaccharide: roles of the receptor complex. Pharmacology \& therapeutics 2003; 100(2): 171-94.

23. Frey E, Miller D, Jahr TG, et al. Soluble CD14 participates in the response of cells to lipopolysaccharide. The Journal of experimental medicine 1992; 176(6): 1665-71.

24. NOCKHER WA, BERGMANN L, SCHERBERICH JE. Increased soluble CD14 serum levels and altered CD14 expression of peripheral blood monocytes in HIV-infected patients. Clinical \& Experimental Immunology 1994; 98(3): 369-74.

25. Gómez-Rial J, Currás-Tuala MJ, Rivero-Calle I, et al. Increased Serum Levels of sCD14 and sCD163 Indicate a Preponderant Role for Monocytes in COVID-19 Immunopathology. Front Immunol 2020; 11: 560381

26. Ziegler-Heitbrock HWL, Ulevitch RJ. CD14: Cell surface receptor and differentiation marker. Immunology Today 1993; 14(3): 121-5.

27. Miyake K. Innate recognition of lipopolysaccharide by CD14 and toll-like receptor 4-MD-2: unique roles for MD-2. International immunopharmacology 2003; 3(1): 119-28.

28. Antal-Szalmas P, van Strijp JAG, Weersink AJL, Verhoef J, van Kessel KPM. Quantitation of surface CD14 on human monocytes and neutrophils. Journal of Leukocyte Biology 1997; 61(6): 721-8.

29. Goyert S, Ferrero E, Rettig W, Yenamandra A, Obata F, Le Beau M. The CD14 monocyte differentiation antigen maps to a region encoding growth factors and receptors. Science 1988; 239(4839): 497-500.

30. Massey HM, Flanagan AM. Human osteoclasts derive from CD14-positive monocytes. British journal of haematology 1999; 106(1): 167-70.

31. Landmann R, Knopf H-P, Link S, Sansano S, Schumann R, Zimmerli W. Human monocyte CD14 is upregulated by lipopolysaccharide. Infection and immunity 1996; 64(5): 1762-9.

32. Lamping N, Dettmer R, Schröder NW, et al. LPS-binding protein protects mice from septic shock caused by LPS or gram-negative bacteria. J Clin Invest 1998; 101(10): 2065-71.

33. Moin ASM, Sathyapalan T, Atkin SL, Butler AE. Letter to the Editor: Pro-fibrotic M2 macrophage markers may increase the risk for COVID19 in type 2 diabetes with obesity. Metabolism: clinical and experimental 2020; 112: 154374-

34. Shive CL, Jiang W, Anthony DD, Lederman MM. Soluble CD14 is a nonspecific marker of monocyte activation. AIDS (London, England) 2015; 29(10): 1263-5. 
35. Iovine NM, Elsbach P, Weiss J. An opsonic function of the neutrophil bactericidal/permeability-increasing protein depends on both its N- and C-terminal domains. Proceedings of the National Academy of Sciences 1997; 94(20): 10973-8.

36. Beamer LJ, Carroll SF, Eisenberg D. The BPI/LBP family of proteins: A structural analysis of conserved regions. Protein Science 1998; 7(4): 906-14.

37. Abrahamson SL, Wu H-M, Williams RE, et al. Biochemical Characterization of Recombinant Fusions of Lipopolysaccharide Binding Protein and Bactericidal/Permeability-increasing Protein: IMPLICATIONS IN BIOLOGICAL ACTIVITY*. Journal of Biological Chemistry 1997; 272(4): 2149-55.

38. Tapping RI, Tobias PS. Cellular Binding of Soluble CD14 Requires Lipopolysaccharide (LPS) and LPS-binding Protein*. Journal of Biological Chemistry 1997; 272(37): 23157-64.

39. Jimenez-Duran G, Luque-Martin R, Patel M, et al. Pharmacological validation of targets regulating CD14 during macrophage differentiation. EBioMedicine 2020; 61: 103039.

40. Pence BD. Severe COVID-19 and aging: are monocytes the key? GeroScience 2020; 42(4): 1051-61.

41. Huang C, Wang Y, Li X, et al. Clinical features of patients infected with 2019 novel coronavirus in Wuhan, China. The lancet 2020; 395(10223): 497-506.

42. Yuki K, Fujiogi M, Koutsogiannaki S. COVID-19 pathophysiology: A review. Clinical immunology 2020: 108427.

43. Pugin J, Ulevitch RJ, Tobias PS. A critical role for monocytes and CD14 in endotoxin-induced endothelial cell activation. Journal of Experimental Medicine 1993; 178(6): 2193-200.

44. Patiño R, Ibarra J, Rodriguez A, et al. Circulating monocytes in patients with diabetes mellitus, arterial disease, and increased CD14 expression. The American Journal of Cardiology 2000; 85(11): 1288-91.

45. Cipolletta C, Ryan KE, Hanna EV, Trimble ER. Activation of Peripheral Blood CD14<sup $>+</$ sup $>$ Monocytes Occurs in Diabetes. Diabetes 2005; 54(9): 2779-86.

46. Brenchley JM, Price DA, Schacker TW, et al. Microbial translocation is a cause of systemic immune activation in chronic HIV infection. Nature medicine 2006; 12(12): 1365-71.

47. van de Weg CA, Koraka P, van Gorp EC, et al. Lipopolysaccharide levels are elevated in dengue virus infected patients and correlate with disease severity. Journal of clinical virology 2012; 53(1): 38-42.

48. Jakubzick CV, Randolph GJ, Henson PM. Monocyte differentiation and antigen-presenting functions. Nature Reviews Immunology 2017; 17(6): 349-62.

49. Zhou Z, Huang C, Zhou Z, et al. Structural insight reveals SARS-CoV-2 ORF7a as an immunomodulating factor for human CD14+ monocytes. Iscience 2021; 24(3): 102187.

50. Morante S, La Penna G, Rossi G, Stellato F. SARS-CoV-2 Virion Stabilization by Zn Binding. Front Mol Biosci 2020; 7: 222-.

51. Zhou Z, Huang C, Zhou Z, et al. Structural insight reveals SARS-CoV-2 ORF7a as an immunomodulating factor for human CD14+ monocytes. iScience 2021; 24(3): 102187.

52. Zhou Y, Fu B, Zheng X, et al. Pathogenic T-cells and inflammatory monocytes incite inflammatory storms in severe COVID-19 patients. National Science Review 2020; 7(6): 998-1002.

53. Nizamudeen ZA, Xu E-R, Karthik V, et al. Structural assessment of SARS-CoV2 accessory protein ORF7a predicts LFA-1 and Mac-1 binding potential. Bioscience Reports 2021; 41(1): BSR20203837.

54. Tan Y-X, Tan TH, Lee MJ-R, et al. Induction of apoptosis by the severe acute respiratory syndrome coronavirus 7a protein is dependent on its interaction with the Bcl-XL protein. Journal of virology 2007; 81(12): 6346-55. 
55. Tan Y-J, Fielding BC, Goh P-Y, et al. Overexpression of 7a, a protein specifically encoded by the severe acute respiratory syndrome coronavirus, induces apoptosis via a caspase-dependent pathway. Journal of virology 2004; 78(24): 14043-7.

56. Yuan $\mathrm{X}, \mathrm{Wu}$ J, Shan Y, et al. SARS coronavirus 7a protein blocks cell cycle progression at G0/G1 phase via the cyclin D3/pRb pathway. Virology 2006; 346(1): 74-85.

57. Kanzawa N, Nishigaki K, Hayashi T, et al. Augmentation of chemokine production by severe acute respiratory syndrome coronavirus $3 \mathrm{a} / \mathrm{X} 1$ and $7 \mathrm{a} / \mathrm{X} 4$ proteins through $\mathrm{NF}-\mathrm{\kappa B}$ activation. FEBS letters 2006; 580(30): 6807-12.

58. Kopecky-Bromberg SA, Martinez-Sobrido L, Palese P. 7a protein of severe acute respiratory syndrome coronavirus inhibits cellular protein synthesis and activates p38 mitogen-activated protein kinase. Journal of virology 2006; 80(2): 785-93.

59. Nelson CA, Pekosz A, Lee CA, Diamond MS, Fremont DH. Structure and intracellular targeting of the SARS-coronavirus Orf7a accessory protein. Structure 2005; 13(1): 75-85.

60. Zhu J-y, Lee J-G, van de Leemput J, Lee H, Han Z. Functional analysis of SARS-CoV-2 proteins in Drosophila identifies Orf6-induced pathogenic effects with Selinexor as an effective treatment. Cell \& Bioscience 2021; 11(1): 59.

61. Nemudryi A, Nemudraia A, Wiegand T, et al. SARS-CoV-2 genomic surveillance identifies naturally occurring truncation of ORF7a that limits immune suppression. Cell Reports 2021: 109197.

62. Cao Z, Xia H, Rajsbaum R, Xia X, Wang H, Shi P-Y. Ubiquitination of SARS-CoV-2 ORF7a promotes antagonism of interferon response. Cellular \& Molecular Immunology 2021; 18(3): 746-8.

63. Gordon DE, Jang GM, Bouhaddou M, et al. A SARS-CoV-2 protein interaction map reveals targets for drug repurposing. Nature 2020; 583(7816): 459-68.

64. Neches RY, Kyrpides NC, Ouzounis CA. Atypical Divergence of SARS-CoV-2 Orf8 from Orf7a within the Coronavirus Lineage Suggests Potential Stealthy Viral Strategies in Immune Evasion. mBio 2021; 12(1): e03014-20.

65. Hänel K, Willbold D. SARS-CoV accessory protein 7a directly interacts with human LFA-1. 2007.

66. Martin-Sancho L, Lewinski MK, Pache L, et al. Functional Landscape of SARS-CoV-2 Cellular Restriction. 2020.

67. Greenfield LK, Whitfield C. Synthesis of lipopolysaccharide O-antigens by ABC transporter-dependent pathways. Carbohydrate research 2012; 356: 12-24.

68. Choi C-H. ABC transporters as multidrug resistance mechanisms and the development of chemosensitizers for their reversal. Cancer Cell Int 2005; 5: 30-.

69. Hänel K, Stangler T, Stoldt M, Willbold D. Solution structure of the X4 protein coded by the SARS related coronavirus reveals an immunoglobulin like fold and suggests a binding activity to integrin I domains. Journal of biomedical science 2006; 13(3): 281-93.

70. Panzera Y, Ramos N, Frabasile S, et al. A deletion in SARS-CoV-2 ORF7 identified in COVID-19 outbreak in Uruguay. Transboundary and Emerging Diseases; $\mathbf{n} / \mathbf{a}(\mathrm{n} / \mathrm{a})$.

71. Saurin W, Hofnung M, Dassa E. Getting in or out: early segregation between importers and exporters in the evolution of ATP-binding cassette (ABC) transporters. Journal of molecular evolution 1999; 48(1): 22-41.

72. Holland IB, Blight MA. ABC-ATPases, adaptable energy generators fuelling transmembrane movement of a variety of molecules in organisms from bacteria to humans. Journal of molecular biology 1999; 293(2): $381-99$. 
73. Smith DJ, Earl AJ, Turner G. The multifunctional peptide synthetase performing the first step of penicillin biosynthesis in Penicillium chrysogenum is a 421,073 dalton protein similar to Bacillus brevis peptide antibiotic synthetases. Embo j 1990; 9(9): 2743-50.

74. Confalonieri F, Duguet M. A 200-amino acid ATPase module in search of a basic function. Bioessays 1995; 17(7): 639-50.

75. Erzberger JP, Berger JM. Evolutionary relationships and structural mechanisms of AAA+ proteins. Annu Rev Biophys Biomol Struct 2006; 35: 93-114.

76. Richard CY, Hanson PI, Jahn R, Brünger AT. Structure of the ATP-dependent oligomerization domain of N-ethylmaleimide sensitive factor complexed with ATP. Nature structural biology 1998; 5(9): 803-11.

77. Iyer LM, Leipe DD, Koonin EV, Aravind L. Evolutionary history and higher order classification of AAA+ ATPases. Journal of structural biology 2004; 146(1-2): 11-31.

78. Hanson PI, Whiteheart SW. AAA+ proteins: have engine, will work. Nature reviews Molecular cell biology 2005; 6(7): 519-29.

79. Connelly JC, Kirkham LA, Leach DR. The SbcCD nuclease of Escherichia coli is a structural maintenance of chromosomes (SMC) family protein that cleaves hairpin DNA. Proceedings of the National Academy of Sciences of the United States of America 1998; 95(14): 7969-74.

80. Hung LW, Wang IX, Nikaido K, Liu PQ, Ames GF, Kim SH. Crystal structure of the ATP-binding subunit of an ABC transporter. Nature 1998; 396(6712): 703-7.

81. Scott JW, Hawley SA, Green KA, et al. CBS domains form energy-sensing modules whose binding of adenosine ligands is disrupted by disease mutations. J Clin Invest 2004; 113(2): 274-84.

82. Pearce SR, Mimmack ML, Gallagher MP, Gileadi U, Hyde SC, Higgins CF. Membrane topology of the integral membrane components, OppB and OppC, of the oligopeptide permease of Salmonella typhimurium. Molecular microbiology 1992; 6(1): 47-57.

83. Aravind L, Koonin EV. The STAS domain - a link between anion transporters and antisigma-factor antagonists. Current biology : $C B$ 2000; 10(2): R53-5.

84. Koonin EV, Wolf YI, Aravind L. Protein fold recognition using sequence profiles and its application in structural genomics. Advances in protein chemistry 2000; 54: 245-75.

85. Higgins CF. ABC transporters: physiology, structure and mechanism--an overview. Res Microbiol 2001; 152(3-4): 205-10.

86. Reizer J, Reizer A, Saier MH, Jr. A new subfamily of bacterial ABC-type transport systems catalyzing export of drugs and carbohydrates. Protein science : a publication of the Protein Society 1992; 1(10): 1326-32.

87. Higgins CF, Gallagher MP, Mimmack ML, Pearce SR. A family of closely related ATP-binding subunits from prokaryotic and eukaryotic cells. Bioessays 1988; 8(4): 111-6.

88. Murina V, Kasari M, Takada H, et al. ABCF ATPases Involved in Protein Synthesis, Ribosome Assembly and Antibiotic Resistance: Structural and Functional Diversification across the Tree of Life. J Mol Biol 2019; 431(18): 3568-90.

89. Deka RK, Brautigam CA, Yang XF, et al. The PnrA (Tp0319; TmpC) lipoprotein represents a new family of bacterial purine nucleoside receptor encoded within an ATP-binding cassette (ABC)-like operon in Treponema pallidum. The Journal of biological chemistry 2006; 281(12): 8072-81.

90. Deka RK, Brautigam CA, Biddy BA, Liu WZ, Norgard MV. Evidence for an ABC-type riboflavin transporter system in pathogenic spirochetes. mBio 2013; 4(1): e00615-12.

91. Janausch IG, Zientz E, Tran QH, Kröger A, Unden G. C4-dicarboxylate carriers and sensors in bacteria. Biochimica et biophysica acta 2002; 1553(1-2): 39-56. 
92. Kelly DJ, Thomas GH. The tripartite ATP-independent periplasmic (TRAP) transporters of bacteria and archaea. FEMS microbiology reviews 2001; 25(4): 405-24.

93. Adams MD, Wagner LM, Graddis TJ, et al. Nucleotide sequence and genetic characterization reveal six essential genes for the LIV-I and LS transport systems of Escherichia coli. The Journal of biological chemistry 1990; 265(20): 11436-43.

94. Kadaba NS, Kaiser JT, Johnson E, Lee A, Rees DC. The high-affinity E. coli methionine ABC transporter: structure and allosteric regulation. Science 2008; 321(5886): 250-3.

95. Friedman DB, Stauff DL, Pishchany G, Whitwell CW, Torres VJ, Skaar EP. Staphylococcus aureus redirects central metabolism to increase iron availability. PLoS Pathog 2006; 2(8): e87.

96. Yakushi T, Masuda K, Narita S, Matsuyama S, Tokuda H. A new ABC transporter mediating the detachment of lipid-modified proteins from membranes. Nat Cell Biol 2000; 2(4): 212-8.

97. Mavrici D, Marakalala MJ, Holton JM, et al. Mycobacterium tuberculosis FtsX extracellular domain activates the peptidoglycan hydrolase, RipC. Proceedings of the National Academy of Sciences of the United States of America 2014; 111(22): 8037-42.

98. Bull PC, Cox DW. Wilson disease and Menkes disease: new handles on heavy-metal transport. Trends in genetics : TIG 1994; 10(7): 246-52.

99. $\mathrm{Xu} \mathrm{Y}, \mathrm{Sim} \mathrm{SH}, \mathrm{Nam} \mathrm{KH}$, et al. Crystal structure of the periplasmic region of MacB, a noncanonic $\mathrm{ABC}$ transporter. Biochemistry 2009; 48(23): 5218-25.

100. An JH, Kim YS. A gene cluster encoding malonyl-CoA decarboxylase (MatA), malonyl-CoA synthetase (MatB) and a putative dicarboxylate carrier protein (MatC) in Rhizobium trifolii--cloning, sequencing, and expression of the enzymes in Escherichia coli. European journal of biochemistry 1998; 257(2): 395-402.

101. Walmsley AR, Barrett MP, Bringaud F, Gould GW. Sugar transporters from bacteria, parasites and mammals: structure-activity relationships. Trends in biochemical sciences 1998; 23(12): 476-81.

102. Madej MG, Dang S, Yan N, Kaback HR. Evolutionary mix-and-match with MFS transporters. Proceedings of the National Academy of Sciences of the United States of America 2013; 110(15): 5870-4.

103. Rodionova IA, Li X, Plymale AE, et al. Genomic distribution of B-vitamin auxotrophy and uptake transporters in environmental bacteria from the Chloroflexi phylum. Environmental microbiology reports 2015; 7(2): 204-10.

104. Kappes RM, Kempf B, Kneip S, et al. Two evolutionarily closely related ABC transporters mediate the uptake of choline for synthesis of the osmoprotectant glycine betaine in Bacillus subtilis. Molecular microbiology 1999; 32(1): 203-16.

105. Saurin W, Dassa E. Sequence relationships between integral inner membrane proteins of binding protein-dependent transport systems: evolution by recurrent gene duplications. Protein science : $a$ publication of the Protein Society 1994; 3(2): 325-44.

106. Vyas NK, Vyas MN, Quiocho FA. The 3 A resolution structure of a D-galactose-binding protein for transport and chemotaxis in Escherichia coli. Proceedings of the National Academy of Sciences of the United States of America 1983; 80(7): 1792-6.

107. Taga ME, Semmelhack JL, Bassler BL. The LuxS-dependent autoinducer AI-2 controls the expression of an $\mathrm{ABC}$ transporter that functions in AI-2 uptake in Salmonella typhimurium. Molecular microbiology 2001; 42(3): 777-93.

108. Singh B, Röhm KH. Characterization of a Pseudomonas putida ABC transporter (AatJMQP) required for acidic amino acid uptake: biochemical properties and regulation by the Aau two-component system. Microbiology (Reading, England) 2008; 154(Pt 3): 797-809. 
109. Magnusson U, Salopek-Sondi B, Luck LA, Mowbray SL. X-ray structures of the leucine-binding protein illustrate conformational changes and the basis of ligand specificity. The Journal of biological chemistry 2004; 279(10): 8747-52.

110. Tam R, Saier MH, Jr. Structural, functional, and evolutionary relationships among extracellular solute-binding receptors of bacteria. Microbiological reviews 1993; 57(2): 320-46.

111. Kuwabara PE, Labouesse M. The sterol-sensing domain: multiple families, a unique role? Trends in genetics : TIG 2002; 18(4): 193-201.

112. Lazarevic V, Karamata D. The tagGH operon of Bacillus subtilis 168 encodes a two-component ABC transporter involved in the metabolism of two wall teichoic acids. Molecular microbiology 1995; 16(2): $345-55$.

113. Roessler D, Hauser U, Wilske B. Heterogeneity of BmpA (P39) among European isolates of Borrelia burgdorferi sensu lato and influence of interspecies variability on serodiagnosis. Journal of clinical microbiology 1997; 35(11): 2752-8.

114. Yakushi T, Masuda K, Narita S, Matsuyama S, Tokuda H. A new ABC transporter mediating the detachment of lipid-modified proteins from membranes. Nat Cell Biol 2000; 2(4): 212-8.

115. Osborn M, Gander J, Parisi E. Mechanism of assembly of the outer membrane of Salmonella typhimurium: site of synthesis of lipopolysaccharide. Journal of Biological Chemistry 1972; 247(12): 3973-86.

116. Pacios Bras C, Jordá MA, Wijfjes AH, et al. A Lotus japonicus nodulation system based on heterologous expression of the fucosyl transferase NodZ and the acetyl transferase NoIL in Rhizobium leguminosarum. Molecular plant-microbe interactions : MPMI 2000; 13(4): 475-9.

117. Yoon JH, Cho HI, Park SS, Chang YH, Kim BK. Mutation analysis of the PIG-A gene in Korean patients with paroxysmal nocturnal haemoglobinuria. J Clin Pathol 2002; 55(6): 410-3.

118. Schulz BL, Jen FE, Power PM, et al. Identification of bacterial protein O-oligosaccharyltransferases and their glycoprotein substrates. PLoS One 2013; 8(5): e62768.

119. Brabetz W, Müller-Loennies S, Brade H. 3-Deoxy-D-manno-oct-2-ulosonic acid (Kdo) transferase (WaaA) and kdo kinase $(\mathrm{KdkA})$ of Haemophilus influenzae are both required to complement a waaA knockout mutation of Escherichia coli. The Journal of biological chemistry 2000; 275(45): 34954-62.

120. Prince C, Jia Z. An Unexpected Duo: Rubredoxin Binds Nine TPR Motifs to Form LapB, an Essential Regulator of Lipopolysaccharide Synthesis. Structure 2015; 23(8): 1500-6.

121. Franco AV, Liu D, Reeves PR. The wzz (cld) protein in Escherichia coli: amino acid sequence variation determines O-antigen chain length specificity. J Bacteriol 1998; 180(10): 2670-5.

122. Beamer LJ, Carroll SF, Eisenberg D. Crystal structure of human BPI and two bound phospholipids at 2.4 angstrom resolution. Science 1997; 276(5320): 1861-4.

123. Weiss J. Bactericidal/permeability-increasing protein (BPI) and lipopolysaccharide-binding protein (LBP): structure, function and regulation in host defence against Gram-negative bacteria. Biochem Soc Trans 2003; 31(Pt 4): 785-90.

124. Kelley SL, Lukk T, Nair SK, Tapping RI. The crystal structure of human soluble CD14 reveals a bent solenoid with a hydrophobic amino-terminal pocket. J Immunol 2013; 190(3): 1304-11.

125. Juan TS, Kelley MJ, Johnson DA, et al. Soluble CD14 truncated at amino acid 152 binds lipopolysaccharide (LPS) and enables cellular response to LPS. The Journal of biological chemistry 1995; 270(3): 1382-7.

126. Viriyakosol S, Kirkland TN. The N-terminal half of membrane CD14 is a functional cellular lipopolysaccharide receptor. Infect Immun 1996; 64(2): 653-6.

127. Petruk G, Puthia M, Petrlova J, Strömdahl A-C, Kjellström S, Schmidtchen A. SARS-CoV-2 Spike protein binds to bacterial lipopolysaccharide and boosts proinflammatory activity. bioRxiv 2020. 
128. Wenzhong L, Hualan L. COVID-19: captures iron and generates reactive oxygen species to damage the human immune system. Autoimmunity 2021; 54(4): 213-24.

129. Caboni M, Pedron T, Rossi O, et al. An O antigen capsule modulates bacterial pathogenesis in Shigella sonnei. PLoS Pathog 2015; 11(3): e1004749.

130. Spanakis N, Kassela K, Dovrolis N, et al. A main event and multiple introductions of SARS-CoV-2 initiated the COVID-19 epidemic in Greece. Journal of Medical Virology 2021; 93(5): 2899-907.

131. Huang C, Ito N, Tseng C-TK, Makino S. Severe acute respiratory syndrome coronavirus 7a accessory protein is a viral structural protein. Journal of virology 2006; 80(15): 7287-94.

132. Addetia A, Xie H, Roychoudhury $\mathrm{P}$, et al. Identification of multiple large deletions in ORF7a resulting in in-frame gene fusions in clinical SARS-CoV-2 isolates. J Clin Virol 2020; 129: 104523-.

133. Lau S-Y, Wang P, Mok BW-Y, et al. Attenuated SARS-CoV-2 variants with deletions at the S1/S2 junction. Emerging microbes \& infections 2020; 9(1): 837-42. 
Table 1. The domains of the ABC system of ORF7a

\begin{tabular}{llcc}
\hline \multicolumn{1}{c}{ Domain } & \multicolumn{1}{c}{ Motif } & Start & End \\
\hline ABC transmembrane & MKIILFLALITLATCELYHYQECVRGTTVLLKEPCSSGTYEGN & 1 & 99 \\
type-1 & SPFHPLADNKFALTCFSTQFAFACPDGVKHVYQLRARSVSPK & & \\
& LFIRQEEVQELYSP & & \\
ABC transporter & MKIILFLALITLATCELYHYQECVRGTTVLLKEPCSSGTYEGN & 1 & 110 \\
& SPFHPLADNKFALTCFSTQFAFACPDGVKHVYQLRARSVSPK & & \\
& LFIRQEEVQELYSPIFLIVAAIVFI & & \\
ABC_tran_Xtn & EPCSSGTYEGNSPFHP & 33 & 48 \\
ABC_transp_aux & YHYQECVRGTTVLLKEPCSSGTYEG & 18 & 42 \\
ABC2_membrane & ATCELYHYQECVRGTTVLLKEPCSSGTYEGNSPFHPLADNKF & 13 & 80 \\
& ALTCFSTQFAFACPDGVKHVYQLRAR & & \\
AAA & CELYHYQECVRGTTVLLKEPCSSGTYEGNSPFHPLADNKFAL & 15 & 91 \\
& TCFSTQFAFACPDGVKHVYQLRARSVSPKLFIRQE & & \\
AAA_15 & HYQECVR & 19 & 25 \\
CBIO & YHYQEC & 18 & 23 \\
& TYEGNSPF & 39 & 46 \\
CBS & YEGNSPFHP & 40 & 48 \\
OppC_N & CELYHYQEC & 15 & 23 \\
STAS & CELYHYQEC & 15 & 23 \\
TOBE_2 & YHYQEC & 18 & 23 \\
& SGTYEGNS & 37 & 44 \\
AMP-binding & NKFALTCFSTQFAFAC & 52 & 67 \\
\hline
\end{tabular}


Table 2. Substances transported by ORF7a's ABC system

\begin{tabular}{|c|c|c|c|c|}
\hline Domain & Substance & Motif & Start & End \\
\hline \multirow[t]{4}{*}{ Bmp } & Purine Nucleoside & CELYHYQECVR & 15 & 25 \\
\hline & /Riboflavin & & & \\
\hline & & NSPFHP & 43 & 48 \\
\hline & & DNKFALTCFSTQFAFACPDGVKHVYQLRARSV & 51 & 82 \\
\hline \multirow[t]{2}{*}{$\mathrm{CBIO}$} & cobalt & YHYQEC & 18 & 23 \\
\hline & & TYEGNSPF & 39 & 46 \\
\hline CitMHS & Citrate/ $\mathrm{Na}+/ \mathrm{H}+$ & YHYQECVR & 18 & 25 \\
\hline \multirow[t]{3}{*}{ DetM } & TRAP & TCELYHYQECVRGTTVLLKEPCSSGTYEGNSPFH & 14 & 102 \\
\hline & C4-dicarboxylate & PLADNKFALTCFSTQFAFACPDGVKHVYQLRARS & & \\
\hline & /periplasmic TRAP & VSPKLFIRQEEVQELYSPIFL & & \\
\hline \multirow[t]{3}{*}{ DUF3382 } & Branched chain & EPCSSGTYEGNSPFHPLADNKFALTCFSTQF & 33 & 63 \\
\hline & amino acid(Leu /Ile & & & \\
\hline & /Val) & & & \\
\hline DUF3708 & methionine & KEPCSSGTYEGNS & 32 & 44 \\
\hline \multirow[t]{4}{*}{ FtsX } & Heme /Lipoprotein & ITLATCELYHYQECV & 10 & 24 \\
\hline & release & & & \\
\hline & & PCSSGTYEGNSPFHPLADNKFALTCFSTQFAFACP & 34 & 90 \\
\hline & & DGVKHVYQLRARSVSPKLFIRQ & & \\
\hline \multirow[t]{2}{*}{ FtsX_ECD } & Heme & YHYQEC & 18 & 23 \\
\hline & & FACPDGVKH & 65 & 73 \\
\hline HMA & Heavy metal & DNKFALTCFSTQFAFACPDGVKHVYQ & 51 & 76 \\
\hline \multirow[t]{3}{*}{ MacB_PCD } & Multi-drug efflux & ATCELYHYQECVRGTTVLLKEPCSSGTYEGNSPF & 13 & 101 \\
\hline & /periplasmic core & HPLADNKFALTCFSTQFAFACPDGVKHVYQLRA & & \\
\hline & & RSVSPKLFIRQEEVQELYSPIF & & \\
\hline MatC_N & Dicarboxylate & YHYQECVRG & 18 & 26 \\
\hline \multirow[t]{2}{*}{ MFS } & Lactose & TCELYHYQECVR & 14 & 25 \\
\hline & & PCSSGTYEGNSPFHPLADNKFALTC & 34 & 58 \\
\hline NIL & D-methionine & CSSGTYEGNSPFHP & 35 & 48 \\
\hline \multirow[t]{3}{*}{ NMT1 } & $\mathrm{VB} 1 / \mathrm{VB} 2$ & ATCELYHYQECVR & 13 & 25 \\
\hline & & NSPFHP & 43 & 48 \\
\hline & & QFAFACPDGVKHVYQLRARSVSPKLFIR & 62 & 89 \\
\hline OppC_N & Oligopeptides & CELYHYQEC & 15 & 23 \\
\hline \multirow[t]{3}{*}{ OpuAC } & Glycine Betaine & YHYQEC & 18 & 23 \\
\hline & Transformation & & & \\
\hline & & CFSTQFAFACPDGVKHV & 58 & 74 \\
\hline \multirow[t]{2}{*}{$\mathrm{PBPb}$} & Periplasmic solute & TCELYHYQECVRGTTVLLKEPCSSGTYEGNSPFH & 14 & 78 \\
\hline & & PLADNKFALTCFSTQFAFACPDGVKHVYQLR & & \\
\hline \multirow[t]{2}{*}{ PDGLE } & metal & YEGNSPFHPLADNKFALTCFSTQFAFACPDGVKH & 40 & 76 \\
\hline & & VYQ & & \\
\hline \multirow[t]{3}{*}{ Peripla_BP_4 } & D-galactose /Ribose & TCELYHYQECVRGTTVLLKEPCSSGTYEGNSPFH & 14 & 59 \\
\hline & /AI-2 /periplasmic & PLADNKFALTCF & & \\
\hline & TRAP & & & \\
\hline
\end{tabular}




\begin{tabular}{|c|c|c|c|c|}
\hline & & HVYQLR & 73 & 78 \\
\hline \multirow[t]{3}{*}{ Peripla_BP_6 } & Branched chain & CELYHYQECVRGTTVLLKEPCSSGTYEGNSPFHP & 15 & 97 \\
\hline & amino acid (Leu /Ile & LADNKFALTCFSTQFAFACPDGVKHVYQLRARS & & \\
\hline & /Val) & VSPKLFIRQEEVQELY & & \\
\hline \multirow[t]{3}{*}{ SBP_bac_5 } & Periplasmic solute & ITLATCELYHYQECVRGTTVLLKEPCSSGTYEGN & 10 & 100 \\
\hline & /heme-binding & SPFHPLADNKFALTCFSTQFAFACPDGVKHVYQL & & \\
\hline & lipoprotein & RARSVSPKLFIRQEEVQELYSPI & & \\
\hline SSD & Sterol & HYQECVRG & 19 & 26 \\
\hline STAS & SLC26A anion & CELYHYQEC & 15 & 23 \\
\hline & /sulfate & & & \\
\hline TAGH & Teichoic acid & ACPDGVKHVYQLR & 66 & 78 \\
\hline \multirow[t]{3}{*}{ TOBE_2 } & Molybdenum & YHYQEC & 18 & 23 \\
\hline & Sulfate & & & \\
\hline & & SGTYEGNS & 37 & 44 \\
\hline
\end{tabular}


Table 3 Related domains of lipopolysaccharide synthesized by ORF7a

\begin{tabular}{|c|c|c|c|c|}
\hline Domain & Synthetic molecule & Motif & Start & End \\
\hline \multirow[t]{3}{*}{ Acyl_transf_3 } & Lipids & YHYQEC & 18 & 23 \\
\hline & & KEPCSSGTYEGNSPFHPLADNKFALTCFSTQF & 32 & 76 \\
\hline & & AFACPDGVKHVYQ & & \\
\hline \multirow[t]{2}{*}{ CTP_transf_like } & Core & YHYQECVRGTTVLLKEPCSSGTYEGNSPFHPL & 18 & 75 \\
\hline & polysaccharide & ADNKFALTCFSTQFAFACPDGVKHVY & & \\
\hline \multirow[t]{2}{*}{ Glyco_transf_8C } & Core & HVYQLRARSVSPK & 73 & 85 \\
\hline & polysaccharide & & & \\
\hline \multirow[t]{4}{*}{ Glycos_transf_1 } & Core & TCELYHYQECVRGTTVLLKEPCSSGTYEGNSP & 14 & 48 \\
\hline & polysaccharide & FHP & & \\
\hline & & ALTCFSTQFAFACPDGVKHVYQLRARSVSPK & 55 & 106 \\
\hline & & LFIRQEEVQELYSPIFLIVAA & & \\
\hline PglL_A & O-specific & KEPCSSGTYEGNSPFH & 32 & 47 \\
\hline & polysaccharide & & & \\
\hline \multirow[t]{4}{*}{ Glycos_transf_N } & Lipopolysaccharide & MKIILF & 1 & 6 \\
\hline & & CELYHYQECVRGTTVLLKEPCSSGTYEGNSPF & 15 & 82 \\
\hline & & HPLADNKFALTCFSTQFAFACPDGVKHVYQL & & \\
\hline & & RARSV & & \\
\hline \multirow[t]{3}{*}{ Rubredoxin_2 } & Lipopolysaccharide & CELYHYQECVRGTTVLLKEPCSSGTYEGNSPF & 15 & 52 \\
\hline & & HPLADN & & \\
\hline & & STQFAFACPDGVKH & 60 & 73 \\
\hline Rubredoxin-like & Lipopolysaccharide & YHYQECVRGTTVLLKEPCSSGTYEGNSPFHP & 18 & 48 \\
\hline \multirow[t]{4}{*}{ Wzz } & Lipopolysaccharide & MKIILFLALITLATCELYHYQECVRGTTVLLK & 1 & 120 \\
\hline & & EPCSSGTYEGNSPFHPLADNKFALTCFSTQFA & & \\
\hline & & FACPDGVKHVYQLRARSVSPKLFIRQEEVQEL & & \\
\hline & & YSPIFLIVAAIVFITLCFTLKRKT & & \\
\hline
\end{tabular}




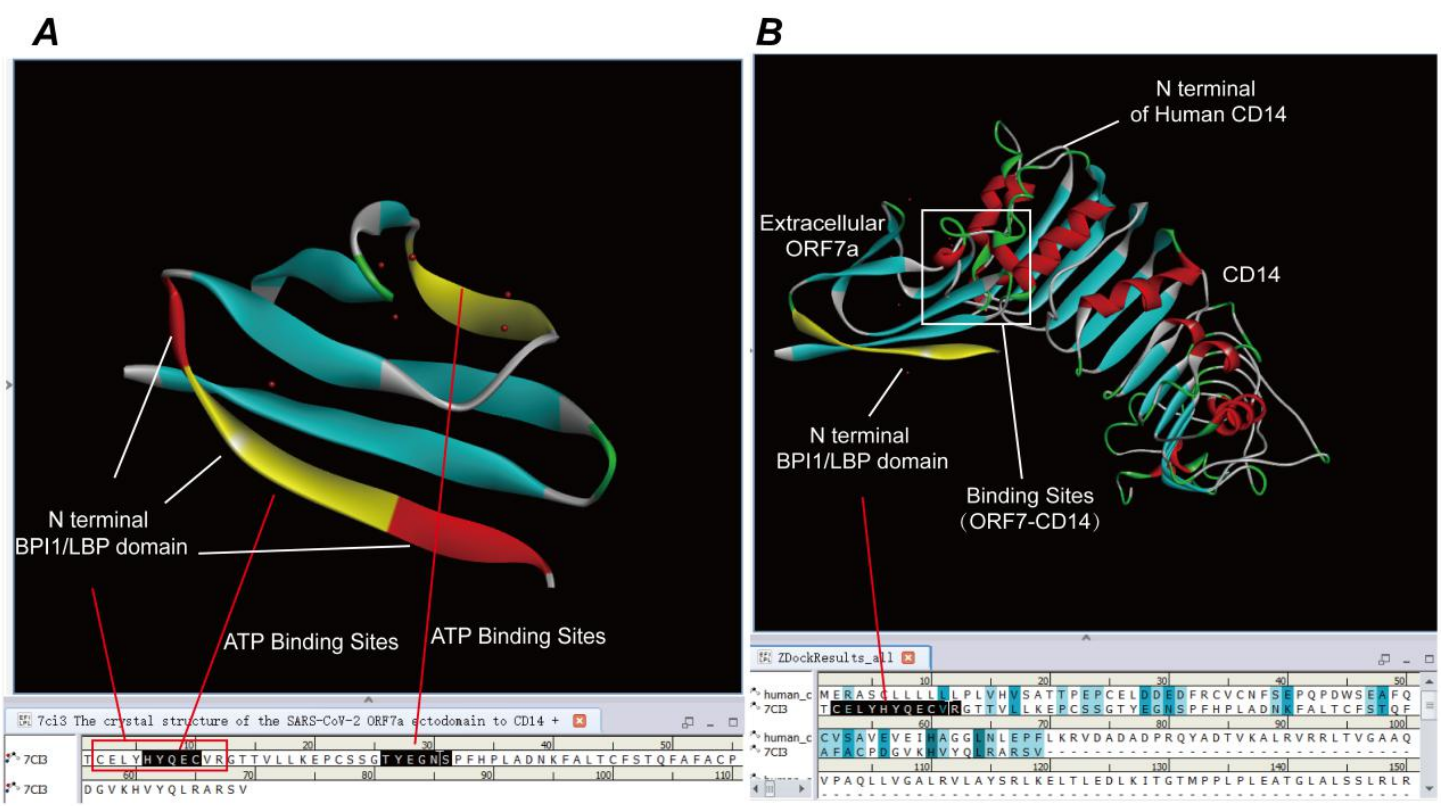

Figure 1. Extracellular ORF7a (PDB ID: 7ci3; 14-96) binds LPS and CD14. A. the BPI1/LBP domain of extracellular ORF7a binds to LPS. ORF7a has two ATP binding sites, "HYQEC" and "TYEGNS" . "HYQEC" is in the BPI1/LBP domain. B. Extracellular ORF7a binds to human CD14 antigen molecules. 